\title{
Vanishing set of inverse Jacobi multipliers and attractor/repeller sets
}

Cite as: Chaos 31, 013113 (2021); https://doi.org/10.1063/5.0020360

Submitted: 01 July 2020 . Accepted: 04 November 2020 . Published Online: 06 January 2021

(iD) Isaac A. García, (D) Jaume Giné, (D) Jaume Llibre, and (D) Susanna Maza

\section{Sign up for topic alerts} New articles delivered to your inbox 


\title{
Vanishing set of inverse Jacobi multipliers and attractor/repeller sets
}

\author{
Cite as: Chaos 31, 013113 (2021); doi: 10.1063/5.0020360 \\ Submitted: 1 July 2020 . Accepted: 4 November 2020 . \\ Published Online: 6 January 2021

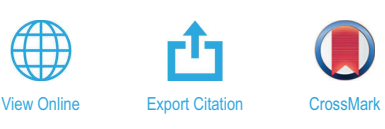

Isaac A. García, ${ }^{1, a)}$ (D) Jaume Giné, , ,b) (D) Jaume Llibre,, ${ }^{2, c)}$ (D) and Susanna Maza ${ }^{1, d)}$ (D)

\author{
AFFILIATIONS \\ ${ }^{1}$ Departament de Matemàtica, Universitat de Lleida, Avda. Jaume II, 69, 25001 Lleida, Catalonia, Spain \\ a) E-mail: garcia@matematica.udl.cat

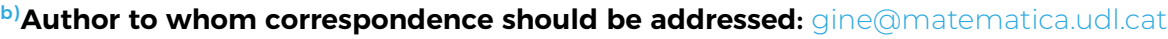 \\ ${ }^{c}$ E-mail: jllibre@mat.uab.cat \\ d)E-mail: smaza@matematica.udl.cat
}

${ }^{2}$ Departament de Matemàtiques, Universitat Autònoma de Barcelona, 08193 Bellaterra, Barcelona, Catalonia, Spain

\begin{abstract}
In this paper, we study conditions under which the zero-set of the inverse Jacobi multiplier of a smooth vector field contains its attractor/repeller compact sets. The work generalizes previous results focusing on sink singularities, orbitally asymptotic limit cycles, and monodromic attractor graphics. Taking different flows on the torus and the sphere as canonical examples of attractor/repeller sets with different topologies, several examples are constructed illustrating the results presented.
\end{abstract}

Published under license by AIP Publishing. https://doi.org/10.1063/5.0020360

The aim of this paper is to study under what restrictions the zero-set of the inverse Jacobi multiplier of a smooth vector field contains its compact attractor or repeller sets. The results presented generalize the previous ones by extending the topology of the invariant set. Different flows on the torus and the sphere are given as canonical examples of attractor/repeller sets with different genus. Also, other additional examples are constructed.

\section{INTRODUCTION AND MAIN RESULTS}

We consider $C^{1}$ autonomous ordinary differential equations $\dot{x}=f(x)$ defined in $\mathbb{R}^{n}$ where $x=\left(x_{1}, \ldots, x_{n}\right) \in \mathbb{R}^{n}$ and its associated vector field $\mathcal{X}=\sum_{i=1}^{n} f_{i}(x) \partial_{x_{i}}$ where $f(x)=\left(f_{1}(x), \ldots, f_{n}(x)\right)$. We denote by $\operatorname{sing}(\mathcal{X})$ the set of singular points of $\mathcal{X}$ and by $\operatorname{div} \mathcal{X}=\sum_{i=1}^{n} \partial f_{i}(x) / \partial x_{i}$ its divergence.

Let $\phi_{t}$ be the flow associated to $\mathcal{X}$ with $\phi_{0}$ being the identity function. A set $\Gamma \subset \mathbb{R}^{n}$ is invariant if $\phi_{t}(\Gamma)=\Gamma$ for any $t$ for which the flow is defined. We define the distance from a point $p \in \mathbb{R}^{n}$ to the set $\Gamma$ as $d(p ; \Gamma)=\inf \{d(p, q): q \in \Gamma\}$, where $d(p, q)$ is the Euclidean distance between the points $p$ and $q$. If $d\left(\phi_{t}(p) ; \Gamma\right) \rightarrow 0$ when $t \rightarrow \infty$ we will write $\phi_{t}(p) \rightarrow \Gamma$ as $t \rightarrow \infty$. The basin of attraction of a set $\Gamma$ is the set $\left\{p \in \mathbb{R}^{n}: \phi_{t}(p) \rightarrow \Gamma\right\}$, that is, the set of initial conditions that approaches to $\Gamma$ asymptotically under the forward flow. The basin of attraction is an open set.

There are several equivalent ways to define an attractor set. In Refs. 13 and 14, Milnor introduced a definition of attractor. A set $\Gamma \subset \mathbb{R}^{n}$ is an attractor set if it possesses a compact neighborhood $U \subset \mathbb{R}^{n}$ and there is a positive increasing time sequence $\left\{t_{n}\right\}_{n \in \mathbb{N}} \subset \mathbb{R}^{+}$with $t_{n} \rightarrow \infty$ such that $\Gamma=\cap_{n \in \mathbb{N}} \phi_{t_{n}}(U)$, where the nested sequence of sets $U \supsetneq \phi_{t_{1}}(U) \supsetneq \phi_{t_{2}}(U) \supsetneq \cdots$ holds, this $U$ is called a trapping neighborhood of $\Gamma$. Their intersection $\Gamma$ is always invariant, $\phi_{t}(\Gamma)=\Gamma$.

We recall that a non-compact set $\Gamma$ such that $\phi_{t}(p) \rightarrow \Gamma$ as $t \rightarrow \infty$ for any point $p \in \mathbb{R}^{n}$ is not necessarily an invariant set. An example with $n=2$ is given by the curve $\Gamma=\left\{(x, y) \in \mathbb{R}^{2}\right.$ : $y=\exp (-x) \sin (x)\}$ and the flow $\phi_{t}(x, y)=(x+t, y \exp (-t))$ associated with the system $\dot{x}=1, \dot{y}=-y$.

There is a simple sufficient condition to guarantee that a compact invariant set $\Gamma$ is indeed an attractor. It is based on the Liouville divergence-flow relation

$$
\left.\frac{d}{d t}\left|\phi_{t}(U)\right|\right|_{t=t_{0}}=\int_{\phi_{t_{0}}(U)} \operatorname{div}(\mathcal{X}) d x
$$

for any Lebesgue measurable subset $U \subset \mathbb{R}^{n}$, see the proof in Proposition 14 of the Appendix. Here, $|A|$ denotes the volume of 
the set $A$. From this expression, it follows easily that if $\phi_{t}(U) \subseteq U$ and $\left.\operatorname{div}(\mathcal{X})\right|_{U}<0$, then the volume of $U$ contracts to zero under the flow, that is, $\left|\phi_{t}(U)\right| \rightarrow 0$ as $t \rightarrow \infty$. In particular, if $U$ is compact, then it is a trapping neighborhood of an attractor set $\Gamma \subset U$ with $|\Gamma|=0$.

A neighborhood $U$ of a subset $\Gamma \subset \mathbb{R}^{n}$ is a subset of $\mathbb{R}^{n}$, which contains an open set containing $\Gamma$. The $\varepsilon$-neighborhood of $\Gamma$ is the set $U_{\varepsilon}(\Gamma)=\cup_{p \in \Gamma} U_{\varepsilon}(p)$, where for any point $p \in \mathbb{R}^{n}, U_{\varepsilon}(p)$ is the open ball centered at $p$ with radius $\varepsilon$.

We say that the orbit through a point $p$ of an invariant set $\Gamma$ is dense if, for any $\varepsilon>0$ and any $q \in \Gamma$, there is a time $\tau$ such that $\phi_{\tau}(p) \in U_{\varepsilon}(q)$. An attractor is an attractor set that contains a dense orbit; that is, the flow is transitive. This extra condition is included to remove the possibility that an attractor be the union of smaller attractor sets. An attractor is minimal if all its orbits are dense.

A repeller, a repeller set, and a basin of repulsion are an attractor, an attractor set and a basin of attraction for $t \rightarrow-\infty$, respectively.

An important tool in the analysis of the dynamics of vector fields $\mathcal{X}$ in $\mathbb{R}^{n}$ is the determination of manifolds embedded in $\mathbb{R}^{n}$, which are invariant under the flow of $\mathcal{X}$. An invariant manifold defined by the zero-set of a $C^{1}$ function $F: \mathbb{R}^{n} \rightarrow \mathbb{R}$ has associated a continuous cofactor $K$ if $\mathcal{X}(F)=K F$ holds. Sign defined cofactors of attractor invariant sets are good tools to localize their basin of attraction as the following theorem shows.

Our main results are the following two theorems.

Theorem 1: Let $\mathcal{X}$ be a $C^{1}$ vector field in $\mathbb{R}^{n}$ generating a flow $\phi_{t}($.$) and having a C^{1}$ invariant manifold $\{F=0\} \subset \mathbb{R}^{n}$ with associated continuous cofactor $K$. Let $\Omega \subset \mathbb{R}^{n}$ be an open set such that $\Omega \cap\{F=0\}=\emptyset, \phi_{t}(\Omega) \subseteq \Omega$, the restriction $\left.F\right|_{\Omega}$ is well defined and the restriction $\left.K\right|_{\Omega}$ is sign defined and uniformly bounded. Then, $\Omega \backslash \operatorname{sing}(\mathcal{X})$ belongs to the basin of attraction or repulsion of $\{F=0\}$ if $\left.K\right|_{\Omega}<0$ or $\left.K\right|_{\Omega}>0$, respectively.

Theorem 1 is proved in Sec. II.

A $\mathcal{C}^{1}$ function $V: \mathcal{N} \subset \mathbb{R}^{n} \rightarrow \mathbb{R}$ non-locally null in $\mathcal{N}$ is said to be an inverse Jacobi multiplier for the vector field $\mathcal{X}$ in $\mathcal{N}$ if $V$ is a solution of the linear first order partial differential equation $\mathcal{X} V=V \operatorname{div} \mathcal{X}$. This kind of functions $1 / V$ were used by Jacobi to find the last additional first integral needed to get complete integrability of $\mathcal{X}$ although currently they have been used to analyze several dynamical properties of $\mathcal{X}$. The special case $n=2$ has been treated in many papers, see Ref. 7 and references therein and some applications in the higher dimensional case $n \geq 3$ have been reported in Refs. 2 and 3. The next theorem relates the zero-set of inverse Jacobi multipliers and the compact attractor/repeller sets and it is a generalization of some results in Refs. 8 and 4 for $n=2$ and in Ref. 1 when $n \geq 2$.

Theorem 2: Consider a $C^{1}$ vector field $\mathcal{X}$ in $\mathbb{R}^{n}$ and let $\Gamma \subset \mathbb{R}^{n}$ be any zero measure compact attractor/repeller set for its associated flow $\phi_{t}$. Let $V$ be any inverse Jacobi multiplier of $\mathcal{X}$ defined in some neighborhood of $\Gamma$. The following statements hold.

(a) There exists a point $p \in \Gamma$ such that $V(p)=0$, hence $V\left(\phi_{t}(p)\right)$ $=0$ for all $t \in \mathbb{R}$.

(b) If $\Gamma$ is a minimal attractor/repeller, then $\Gamma \subset V^{-1}(0)$.

Theorem 2 is proved in Sec. II.

We remark that the proof of Theorem 2 is essentially different from the one given in Theorem 17 of Ref. 1 for the special case when $\Gamma$ is an asymptotically orbitally stable limit cycle. Our method is more general because it is based on the saturation under the flow of a (global) trapping neighborhood of $\Gamma$, while the proof of Ref. 1 needs the saturation of just a neighborhood of a point of $\Gamma$ under the extra assumption that $|\Gamma|=0$. The reader may consult Ref. 12 to see how different stability definition are related between them.

We recall that an invariant set $\Gamma$ is orbitally stable if for any neighborhood $W \subset \mathbb{R}^{n}$ of $\Gamma$ there exists another neighborhood $U \subset W$ of $\Gamma$ such that $\phi_{t}(U) \subset W$. Moreover, $\Gamma$ is orbitally asymptotically stable if it is orbitally stable and there is a neighborhood $U$ of $\Gamma$ such that for any point $p \in U$ it follows that $d\left(\phi_{t}(p) ; \Gamma\right) \rightarrow 0$ when $t \rightarrow \infty$. The next proposition proves why our approach is more general than the one given in Ref. 1 .

Proposition 3: Let $\Gamma$ be a compact invariant set orbitally asymptotically stable for a flow $\phi_{t}$. Then, $\Gamma$ possesses a trapping neighborhood.

Proposition 3 is proved in Sec. II.

The paper is organized as follows. Section II is dedicated to prove our main results. In Sec. III, we show some nontrivial applications of the presented theory focusing on flows with invariant tori and spheres, while in Sec. IV we consider other examples.

\section{PROOF OF THE MAIN RESULTS}

Proof of Theorem 1. The first step furnishes an expression [see Eq. (2)] for $F$ along the orbits of the system. This formula, although simple, will provide the main tool to prove the theorem. Using the method of characteristics, we can integrate the expression $\mathcal{X}(F)$ $=K F$ along the regular orbit of $\mathcal{X}$ through any point $p \in \mathbb{R}^{n}$ with $p \notin \operatorname{sing}(\mathcal{X})$ in some time interval. This can be achieved by realizing that

$$
\left.\frac{d}{d t}\left(\left(F \circ \phi_{t}\right)(p)\right)=\left(\mathcal{X}(F) \circ \phi_{t}\right)(p)=(K F) \circ \phi_{t}\right)(p) .
$$

The outcome is that we get

$$
\left(F \circ \phi_{t}\right)(p)=F(p) \exp \left(\int_{0}^{t}\left(K \circ \phi_{\tau}\right)(p) d \tau\right) .
$$

We consider any point $p \in \Omega \backslash \operatorname{sing}(\mathcal{X})$ and let $\kappa$ be the uniform bound of the cofactor $K$ in $\Omega$, that is, $|K(x)| \leq \kappa$ for any $x \in \Omega$. From (2) and the fact that $\phi_{t}(\Omega) \subseteq \Omega$, it follows that

$$
\begin{aligned}
\left|F\left(\phi_{t}(p)\right)\right| & =|F(p)| \exp \left(\int_{0}^{t} K\left(\phi_{\tau}(p)\right) d \tau\right) \\
& \leq\left\{\begin{array}{ccc}
|F(p)| \exp (\kappa t) & \text { if } & \left.K\right|_{\Omega}>0, \\
|F(p)| \exp (-\kappa t) & \text { if } & \left.K\right|_{\Omega}<0 .
\end{array}\right.
\end{aligned}
$$

Therefore, $\lim _{t \rightarrow \pm \infty}\left|F\left(\phi_{t}(p)\right)\right|=0$ according to whether $\left.K\right|_{\Omega}<0$ or $\left.K\right|_{\Omega}>0$, respectively. This proves the theorem.

Remark 4: Theorem 1 in the particular case that $\Omega=\mathbb{R}^{n} \backslash\{F=0\}$ implies that $\{F=0\}$ is a global attractor or repeller set according to whether $\left.K\right|_{\Omega}<0$ or $\left.K\right|_{\Omega}>0$, respectively.

Remark 5: The Cauchy-Kovalevskaya theorem ${ }^{5}$ guarantees the existence of a local analytic solution $F$ around any given nonsingular point of an analytic vector field $\mathcal{X}$ of the partial differential equation $\mathcal{X}(F)=K F$ with a fixed $K$. But, Theorem 1 needs that $F$ and $K$ be globally defined on $\Omega$, see, for example, (2). A very important particular case consists in the class of polynomial vector fields of 
degree $m$ and its eventual invariant algebraic variety $\{F=0\}$. Note that in this particular case the associated cofactors $K$ are also polynomials and its degree is bounded by $m-1$, hence the constant cofactors are natural candidates to apply Theorem 1.

Proof of Theorem 2. We prove the theorem assuming that $\Gamma$ is a compact attractor set, a similar proof can be done if $\Gamma$ is a repeller set.

Given a Lebesgue measurable set $D \subset \mathbb{R}^{n}$, we denote by $|D|$ its volume. Given any fixed measurable set $D \subset \mathcal{N} \backslash V^{-1}(0)$, we define the integral invariant as the volume integral

$$
I(t ; D)=\int_{\phi_{t}(D)} \frac{1}{V} d x .
$$

If $V$ is an inverse Jacobi multiplier of the vector field $\mathcal{X}$ in $\mathcal{N} \subset \mathbb{R}^{n}$, then its zero-set $V^{-1}(0)=\{p \in \mathcal{N} \mid V(p)=0\}$ is composed of orbits of $\mathcal{X}$ in $\mathcal{N}$. Indeed for any point $p \in \mathcal{N}$, denoting by $\phi_{t}$ the flow of $\mathcal{X}$, it follows as in (2) that

$$
V\left(\phi_{t}(p)\right)=V(p) \exp \left(\int_{0}^{t} \operatorname{div} \mathcal{X} \circ \phi_{s}(p) d s\right) .
$$

In particular, if $V(p)=0$, then $V\left(\phi_{t}(p)\right)=0$ for all $t$.

Let $\mathcal{N} \subset \mathbb{R}^{n}$ be a neighborhood of $\Gamma$ such that $\left.V\right|_{\mathcal{N}}$ is defined. Let $U$ be trapping neighborhood of $\Gamma$, hence $U$ is compact and there is a positive increasing time sequence $\left\{t_{n}\right\}_{n \in \mathbb{N}} \subset \mathbb{R}^{+}$with $t_{n} \rightarrow \infty$ such that

$$
\Gamma=\bigcap_{n \in \mathbb{N}} \phi_{t_{n}}(U)
$$

with

$$
U \supsetneq \phi_{t_{1}}(U) \supsetneq \phi_{t_{2}}(U) \supsetneq \cdots \cdots
$$

In particular, $\Gamma$ is an invariant set. Without loss of generality, we may assume that $U \subset \mathcal{N}$.

If $V$ vanishes at any point $q \in U \backslash \Gamma$, then from (3) $V$ vanishes on all the orbit $\phi_{t}(q)$ for any $t>0$ and by the continuity of $V, V$ vanishes in at least one point of $\Gamma$, and consequently if $\Gamma$ is a minimal attractor $V$ vanishes in all $\Gamma$. So, we assume the above does not happen, that is, that $\left.V\right|_{U \backslash \Gamma} \neq 0$.

Under our assumptions, the volume $\left|\phi_{t}(U)\right|$ is defined for all $t \geq 0$ and, since $\Gamma$ is compact and has zero measure, $\left|\phi_{t}(U)\right| \rightarrow 0$ as $t \rightarrow \infty$.

We consider the compact set $D=\left\{\phi_{t}(p) \mid p \in \partial U\right.$ and $0 \leq t$ $\left.\leq t_{1}\right\} \subset U$ and the integral invariant

$$
I(t ; D)=\int_{\phi_{t}(D)} \frac{1}{V} d x .
$$

For any fixed $t \geq 0$, by the Mean Value Theorem for Integrals, there exists a point $p(t) \in \phi_{t}(D)$ such that

$$
I(t ; D)=\frac{1}{V(p(t))}\left|\phi_{t}(D)\right| .
$$

Clearly, $\left|\phi_{t}(D)\right|$ is defined for all $t \geq 0$ and $\left|\phi_{t}(D)\right| \rightarrow 0$ as $t \rightarrow \infty$, hence $1 / V(p(t)) \rightarrow \infty$ as $t \rightarrow \infty$, because $I(t ; D)$ is independent of $t$. We conclude that $V(p(t)) \rightarrow 0$ and since $p(t) \rightarrow p \in \Gamma$, by continuity of $V, V(p)=0$ proving statement (a).
Under the hypothesis of (b), $\Gamma$ is, moreover, a minimal attractor. Then, considering again the point $p \in \Gamma$ with $V(p)=0$ predicted by statement (a), its associated orbit $\gamma_{p}=\left\{\phi_{t}(p): t \in \mathbb{R}\right\}$ is dense on $\Gamma$. Recall that $\gamma_{p} \subset V^{-1}(0)$ from (3), so by the continuity of $V$ we claim that $\Gamma \subset V^{-1}(0)$. This last assertion follows taking into account the topological considerations that the zero-set of a continuous function is a closed set (the pre-image of the closed set $\{0\}$ ) and that the topological closure of a dense subset is the whole set.

Proof of Proposition 3. Let $U_{1} \subset \mathbb{R}^{n}$ be a sufficiently small compact neighborhood of the orbitally asymptotically stable set $\Gamma$. Then, for any point $p \in \partial U_{1}$ on the boundary of $U_{1}$, there is a point $p^{*}(p) \in \partial U_{1}$ and a time $\tau(p) \geq 0$ such that $\phi_{\tau(p)}(p)=p^{*}(p)$ $\in \partial U_{1}$ and $\phi_{t}\left(p^{*}\right) \subset U_{1} \backslash \partial U_{1}$ for all $t>0$. Since $U_{1}$ is compact, the function $\tau: \partial U_{1} \rightarrow \mathbb{R}$ is bounded and moreover it is a continuous function by the theorem of continuous dependence of solutions on initial conditions. Hence, defining $0 \leq T_{1}=\max _{p \in \partial U_{1}}\{\tau(p)\}$, it follows that $\Gamma \subset \phi_{t}\left(U_{1}\right) \subsetneq U_{1}$ for any $t>T_{1}$. We define $U_{2}=\phi_{\tau_{1}}\left(U_{1}\right)$ for some fixed $\tau_{1}>T_{1}$. Repeating the above arguments, we construct a sequence $\left\{U_{j}\right\}_{j \in \mathbb{N}} \subset \mathbb{R}^{n}$ of compact neighborhoods of $\Gamma$ and a sequence $\left\{\tau_{j}\right\}_{j \in \mathbb{N}} \subset \mathbb{R}$ of positive times such that $\Gamma \subset U_{j}$ and $\phi_{\tau_{j}}\left(U_{j}\right):=U_{j+1} \subsetneq U_{j}$ for any $j$. As a result, defining the time sequence $\left\{t_{j}\right\}_{j \in \mathbb{N}} \subset \mathbb{R}$ as $t_{j}=\sum_{i=1}^{j} \tau_{i}$ and taking $U=U_{1}$ we may rewrite the former as $U \supsetneq \phi_{t_{1}}(U) \supsetneq \phi_{t_{2}}(U) \supsetneq \cdots$, which implies that $U$ is a trapping neighborhood of $\Gamma$.

\section{SOME APPLICATIONS}

\section{A. Flows with invariant torus}

We consider (embedded in $\mathbb{R}^{3}$ ) a two-dimensional torus $\mathbb{T}^{2}=\mathbb{S}^{1} \times \mathbb{S}^{1}$. In Cartesian coordinates, we describe the torus centered at the origin by $\mathbb{T}^{2}=\{F=0\} \subset \mathbb{R}^{3}$ with $F(x, y, x)$ $=\left(R-\sqrt{x^{2}+y^{2}}\right)^{2}+z^{2}-r^{2}$, where $R$ is the radius from the origin to the center of the generating torus, and $r$ is the radius of the torus with $r<R$, see Fig. 1 .

A local curvilinear coordinate system $(\rho, \theta, \varphi)$ well adapted to the solid torus with boundary $\mathbb{T}^{2}$ is defined by

$$
x=(R+\rho \cos \theta) \cos \varphi, \quad y=(R+\rho \cos \theta) \sin \varphi, \quad z=\rho \sin \theta,
$$

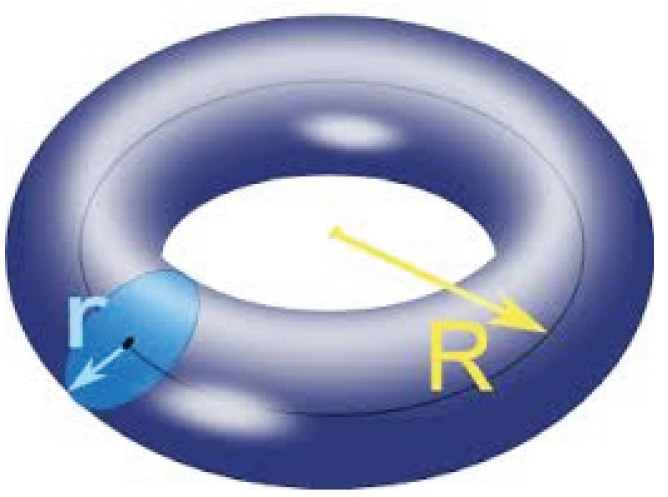

FIG. 1. The two generating radii of $\mathbb{T}^{2}$. 
with $0 \leq \rho \leq r$ and $(\theta, \varphi) \in \mathbb{S}^{1} \times \mathbb{S}^{1}$ being $\mathbb{S}^{1}=\mathbb{R} / 2 \pi \mathbb{Z}$. In these coordinates, $\mathbb{T}^{2}=\{\rho=r\}$ and the points of the torus are parameterized by the angles $(\theta, \varphi)$. Therefore, a vector field generating a flow on $\mathbb{T}^{2}$ is given by $\dot{\theta}=A(\theta, \varphi), \dot{\varphi}=B(\theta, \varphi)$, where $A$ and $B$ are $C^{1}$ functions defined on $\mathbb{T}^{2}$, hence $2 \pi$-periodic in both variables.

Using these coordinates, we analyze the zero-set of the inverse Jacobi multipliers of a family of decoupled three-dimensional analytic vector fields $\mathcal{X}$ having invariant attractor $\mathbb{T}^{2}$ and such that the restricted vector field $\left.\mathcal{X}\right|_{\mathbb{T}^{2}}$ satisfies the hypothesis of Kolmogorov's theorem (see Ref. 15) for the flows on the torus.

Proposition 6: Consider the decoupled analytic system

$$
\begin{aligned}
& \dot{\rho}=(r-\rho) f(\rho, \theta, \varphi), \\
& \dot{\theta}=g(\theta, \varphi), \\
& \dot{\varphi}=h(\theta, \varphi),
\end{aligned}
$$

with $f(r, \theta, \varphi)>0, g^{2}+h^{2}>0$ and where the restricted system to the invariant torus $\mathbb{T}^{2}=\{\rho=r\}$ possesses an analytic invariant Lebesgue measure with positive density. Then, $\mathbb{T}^{2}$ is an attractor set, and if there is one analytic inverse Jacobi multiplier $V$ of (5) such that $\mathbb{T}^{2} \subset V^{-1}(0)$, then the zero-set of any other analytic inverse Jacobi multiplier (5) defined in a neighborhood of $\mathbb{T}^{2}$ must contain $\mathbb{T}^{2}$.

Proof. System (5) possesses the invariant torus $\mathbb{T}^{2}=\{\rho=r\}$ with associated cofactor $-f(\rho, \theta, \varphi)$. Hence, if $f(r, \theta, \varphi)>0$ for any $(\theta, \varphi) \in \mathbb{T}^{2}$, then $\mathbb{T}^{2}$ is an attractor by simple inspection of the first component of the field (5) or by Theorem 1 . The restricted vector field $\left.\mathcal{X}\right|_{\mathbb{T}^{2}}=g(\theta, \varphi) \partial_{\theta}+h(\theta, \varphi) \partial_{\varphi}$ satisfies the hypothesis of Kolmogorov's theorem for flows on the torus, that is, is analytic, fixed-point-free $\left(g^{2}+h^{2}>0\right)$, and possesses an analytic invariant measure with positive density. Then, Kolmogorov's theorem (see Ref. 15) assures the existence of an analytic diffeomorphism $\xi$ on $\mathbb{T}^{2}$ which transforms $\left.\mathcal{X}\right|_{\mathbb{T}^{2}}$ into the orbitally linearizable vector field $\xi_{*}\left(\left.\mathcal{X}\right|_{\mathbb{T}^{2}}\right)=A(\theta, \varphi)\left(\partial_{\theta}+\omega \partial_{\varphi}\right)$ with $A(\theta, \varphi)>0$ and $\omega \in \mathbb{R}$. In particular, the diffeomorphism $(\rho, \theta, \varphi) \mapsto(\rho, \xi(\theta, \varphi))$ and the time rescaling dividing the field by $A(\theta, \varphi)$ transform (5) into

$$
\dot{\rho}=(r-\rho) \hat{f}(\rho, \theta, \varphi), \quad \dot{\theta}=1, \quad \dot{\varphi}=\omega,
$$

where $\hat{f}(\rho, \theta, \varphi)=f(\rho, \xi(\theta, \varphi)) / A(\theta, \varphi)$. By hypothesis, there is an analytic inverse Jacobi multiplier $V$ of $(6)$ such that $\mathbb{T}^{2} \subset V^{-1}(0)$. We will prove (by contradiction) that then there is no analytic inverse Jacobi multiplier $\hat{V}$ such that $\mathbb{T}^{2} \not \subset \hat{V}^{-1}(0)$. The case $\omega \notin \mathbb{Q}$ is trivial by Theorem 2(b) because $\mathbb{T}^{2}$ is a minimal attractor of (6) so we restrict ourselves to the remaining case $\omega \in \mathbb{Q}$ where $\mathbb{T}^{2}$ is foliated by periodic orbits.

We define $\Sigma=\mathbb{T}^{2} \cap \hat{V}^{-1}(0)$ that is either empty or composed by a union of periodic orbits of (6) on $\mathbb{T}^{2}$. We recall that a real analytic function of several variables that vanishes on a set of positive measure must be identically zero. Then, if $\Sigma$ has a positive measure and the function $\left.\hat{V}\right|_{\mathbb{T}^{2}}$ is analytic, then it must be identically zero and therefore we reach the contradiction $\mathbb{T}^{2} \subset \hat{V}^{-1}(0)$. Hence, $\Sigma$ must be a zero-measure set on $\mathbb{T}^{2}$.

Notice that if $\hat{V}$ exists then $H=V / \hat{V}$ would be a non-trivial first integral of (6) well defined in $\mathbb{T}^{2} \backslash \Sigma$ and satisfying

$$
\mathbb{T}^{2} \backslash \Sigma \subset H^{-1}(0) .
$$

Since $\mathbb{T}^{2} \not \subset \hat{V}^{-1}(0)$, there exists at least one periodic orbit $\mathcal{C} \subset \mathbb{T}^{2}$ of (6) such that $\mathcal{C} \not \subset \Sigma$. The flow on the torus is given by $\theta\left(t ; \theta_{0}\right)=\theta_{0}+t(\bmod 2 \pi), \varphi\left(t ; \varphi_{0}\right)=\varphi_{0}+\omega t(\bmod 2 \pi)$. Then, $\mathcal{C}=\left\{\left(r, \theta\left(t ; \theta_{0}\right), \varphi\left(t ; \varphi_{0}\right)\right): 0 \leq t<T\right\}$, where $T=2 \pi q$ is the minimal period when $\omega=p / q$ is irreducible.

We will see that there is no (non-trivial) first integral of (6) well defined in $\mathbb{T}^{2} \backslash \Sigma$ and satisfying (7). We denote by $\rho\left(t ;\left(\rho_{0}, \theta_{0}, \varphi_{0}\right)\right)$ the first component of the flow associated with (6), there is $r^{*}>0$ such that $\rho\left(t ;\left(\rho_{0}, \theta_{0}, \varphi_{0}\right)\right) \rightarrow r$ as $t \rightarrow \infty$ for any $r-r^{*}<\rho_{0}<r$ $+r^{*}$, because $\mathbb{T}^{2}$ is an attractor. Hence, the stable manifold $W_{\mathcal{C}}^{s} \subset \mathbb{R}^{3} \quad$ of $\mathcal{C}$ satisfies $W_{\mathcal{C}}^{s} \supset \mathcal{M}_{\mathcal{C}}=\left\{\left(\rho_{0}, \theta\left(t ; \theta_{0}\right), \varphi\left(t ; \varphi_{0}\right)\right)\right.$ : $\left.r-r^{*}<\rho_{0}<r+r^{*}, 0 \leq t<T\right\}$, where $\mathcal{M}_{\mathcal{C}}$ is a two-dimensional manifold such that $\mathcal{M}_{\mathcal{C}} \cap \mathbb{T}^{2}=\mathcal{C}$. The planar restricted vector field $\left.\mathcal{X}\right|_{\mathcal{M}_{\mathcal{C}}}$ has the limit cycle $\mathcal{C}$. If there was one first integral $H$ of $(6)$ well defined in $\mathbb{T}^{2} \backslash \Sigma$, then its restriction $\left.H\right|_{\mathcal{M}_{\mathcal{C}}}$ would be either a constant or a (nontrivial) first integral of $\left.\mathcal{X}\right|_{\mathcal{M}_{\mathcal{C}}}$ near $\mathcal{C}$. The last case produces clearly a contradiction because there is no first integral near a limit cycle in a planar system. So, we continue assuming the first option, that is, $\left.H\right|_{\mathcal{M}_{\mathcal{C}}}$ is a constant. Then, for any $\mathcal{C} \not \subset \Sigma$, $\left.H\right|_{\mathcal{M}_{\mathcal{C}}}=\left.H\right|_{\mathcal{C}}=0$ where the last equality follows from (7), hence $\left.H\right|_{\mathbb{T}^{2} \backslash \Sigma}=0$. In summary, defining

$$
\mathcal{M}=\bigcup_{\mathcal{C} \subset \mathbb{T}^{2} \backslash \Sigma} \mathcal{M}_{\mathcal{C}} \subset \mathbb{R}^{3},
$$

we have proved that $\left.H\right|_{\mathcal{M}}=0$. We conclude that $H \equiv 0$ in a neighborhood of $\mathbb{T}^{2}$ because the measure of $\Sigma$ is zero and therefore $\mathcal{M}$ has positive measure in $\mathbb{R}^{3}$. This implies that $\hat{V}$ cannot exists. $\square$

Remark 7: In the particular case that $\hat{f}$ only depends on $\rho$, system (6) has the inverse Jacobi multiplier $V=(r-\rho) \hat{f}(\rho)$ where clearly $\mathbb{T}^{2} \subset V^{-1}(0)$ holds. If, moreover, we take $\hat{f}(\rho) \equiv 1$, then the linear vector field (6) has the first integral $H=\cos (\varphi-\omega \theta)$ well defined in $\mathbb{R}^{3}$ when $\omega \in \mathbb{Z}$ although condition (7) does not hold.

Given a $C^{1}$ vector field $\mathcal{X}$, we say that a compact attractor set $\Gamma \subset \mathbb{R}^{n}$ is monodromic if there is a compact transversal section $\Sigma$ to the flow with $\Sigma \cap \Gamma \neq \emptyset$ and $\Sigma \subset U$, where $U$ is a trapping neighborhood of $\Gamma$, where a Poincaré map $\Pi: \Sigma \rightarrow \Sigma$ is defined. Moreover, if

$$
\Pi(\Sigma) \subseteq \operatorname{Int}(\Sigma)
$$

where $\operatorname{Int}(\Sigma)$ denotes the set of all interior points of $\Sigma$, then $\Sigma$ is called a trapping region for $\Pi$. Applying $\Pi$ to both sides of (8) and taking into account that $\Pi$ is a diffeomorphism, hence $\Pi(\operatorname{Int}(\Sigma))$ $=\operatorname{Int}(\Pi(\Sigma))$, and it follows that the iterates of $\Pi$ satisfy $\Pi^{n}(\Sigma)$ $\subseteq \operatorname{Int}\left(\Pi^{n-1}(\Sigma)\right)$ for any $n \in \mathbb{N}$. This means that

$$
\Sigma \cap \Gamma=\bigcap_{n \in \mathbb{N}} \Pi^{n}(\Sigma)
$$

We concentrate on the particular case that $\Gamma=\mathbb{T}^{2}$ (diffeomorphically) and that $\Sigma \cap \Gamma=\mathbb{S}^{1}$ (diffeomorphically) is a transversal section of the flow on $\mathbb{T}^{2}$ generated by the vector field $\mathcal{Y}=\mathcal{X}_{\mathbb{T}^{2}}$ $=A(\theta, \varphi) \partial_{\theta}+B(\theta, \varphi) \partial_{\varphi}$. Notice that for those vector fields $\mathcal{Y}$ having no singular points and such that $B>0$, we may consider the 
differential equation

$$
\frac{d \theta}{d \varphi}=\mathcal{F}(\theta, \varphi)
$$

with $\mathcal{F}=A / B$ and $\Sigma=\{(\theta, \varphi): \varphi=0\} \subset \mathbb{T}^{2}$ being the parameterization of the cross section. Thus, we consider its Poincaré map $\hat{\Pi}=\left.\Pi\right|_{\mathbb{T}^{2}}: \mathbb{S}^{1} \rightarrow \mathbb{S}^{1}$ defined as $\hat{\Pi}\left(\theta_{0}\right)=\theta\left(2 \pi ; \theta_{0}\right)$ being $\theta\left(\varphi ; \theta_{0}\right)$ the solution of (9) with initial condition $\theta\left(0 ; \theta_{0}\right)=\theta_{0}$. Clearly, $\hat{\Pi}$ is an orientation preserving diffeomorphism on the circle because orbits on $\mathbb{T}^{2}$ do not intersect. Consequently, without the loss of generality, we have

$$
\hat{\Pi}^{\prime}(\theta)>0 \text { for all } \theta \in \mathbb{S}^{1},
$$

where the prime means derivative with respect to $\theta$. Any periodic orbit $\phi_{t}(p)$ with $p=\left(\theta^{*}, 0\right) \in \mathbb{S}^{1} \subset \mathbb{T}^{2}$ will correspond with a fixed point of some iterate of $\hat{\Pi}$, that is, there is $k \in \mathbb{N}$ such that $\hat{\Pi}^{k}\left(\theta^{*}\right)=\theta^{*}$. If we deal with a family of vector fields $\mathcal{X}_{\lambda}$ depending on some parameters $\lambda$ and $\mathbb{T}^{2}$ persists as invariant manifold under small parameter variations (for example, under normally hyperbolicity conditions), then the resulting Poincaré map $\hat{\Pi}_{\lambda}$ changes its orbit structure. To understand these bifurcations, the rotation number of $\hat{\Pi}_{\lambda}$ is introduced. Recall that (13) restricted to $\mathbb{T}^{2}$ has a periodic orbit if and only if the rotation number

$$
\begin{aligned}
\operatorname{Rot}(\hat{\Pi}) & =\lim _{n \rightarrow \infty} \frac{\tilde{\Pi}^{n}(\theta)-\theta}{n} \\
& =\lim _{k \rightarrow \infty} \frac{1}{2 \pi} \frac{a(\theta)+a(\hat{\Pi}(\theta))+\cdots+a\left(\hat{\Pi}^{k-1}(\theta)\right)}{k}
\end{aligned}
$$

of $\hat{\Pi}$ is a rational number, see, for example, Ref. 15, where $\tilde{\Pi}$ : $\mathbb{R} \rightarrow \mathbb{R}$ is a lift of $\hat{\Pi}$ and $\hat{\Pi}(\theta)=\theta+a(\theta)$.

We can prove the following general result.

Theorem 8: Consider a $C^{1}$ vector field $\mathcal{Y}$ on a manifold diffeomorphic to a torus $\mathbb{T}^{2}$ having a cross section diffeomorphic to $\mathbb{S}^{1}$ and a rational rotation number. Then, any inverse Jacobi multiplier of $\mathcal{Y}$ must vanish on all the periodic orbits of $\mathcal{Y}$.

Proof. If a periodic orbit of $\mathcal{Y}=A(\theta, \varphi) \partial_{\theta}+B(\theta, \varphi) \partial_{\varphi}$ passes through a point $(\theta, \varphi)=\left(\theta^{*}, 0\right) \in \mathbb{S}^{1} \subset \mathbb{T}^{2}$, then there is some $k \in \mathbb{N}$ with $\hat{\Pi}^{k}\left(\theta^{*}\right)=\theta^{*}$. Let $\hat{v}(\theta, \varphi)$ be the inverse Jacobi multiplier of $\mathcal{Y}$, that is, $\mathcal{Y}(\hat{v})=\hat{v} \operatorname{div}(\mathcal{Y})$. Then, the function $v(\theta, \varphi)$ $=\hat{v}(\theta, \varphi) / B(\theta, \varphi)$ is also an inverse Jacobi multiplier of (9), that is, it satisfies the partial differential equation

$$
\frac{\partial v}{\partial \varphi}+\frac{\partial v}{\partial \theta} \mathcal{F}(\theta, \varphi)=\frac{\partial \mathcal{F}}{\partial \theta}(\theta, \varphi) v(\theta, \varphi) .
$$

Adapting Theorem 3 of Ref. 6 to the differential equation (9) on $\mathbb{T}^{2}$, it follows the fundamental relation between the Poincaré map $\hat{\Pi}$ associated with the flow of $\mathcal{Y}$ with cross section $\mathbb{S}^{1}$ and the inverse Jacobi multiplier $v(\theta, \varphi)$ of $(9)$, which is

$$
v(\hat{\Pi}(\theta), 0)=v(\theta, 0) \hat{\Pi}^{\prime}(\theta)
$$

for any $\theta \in \mathbb{S}^{1}$. Clearly, this equation can be rewritten as

$$
v\left(\hat{\Pi}^{j}(\theta), 0\right)=v\left(\hat{\Pi}^{j-1}(\theta), 0\right) \hat{\Pi}^{\prime}\left(\Pi^{j-1}(\theta)\right)
$$

for any $j \in \mathbb{N}$. Using this fact, if $\hat{\Pi}^{k}\left(\theta^{*}\right)=\theta^{*}$ for some $k$, then

$$
\begin{aligned}
v\left(\theta^{*}, 0\right) & =v\left(\hat{\Pi}^{k}\left(\theta^{*}\right), 0\right)=v\left(\hat{\Pi}^{k-1}\left(\theta^{*}\right), 0\right) \hat{\Pi}^{\prime}\left(\Pi^{k-1}\left(\theta^{*}\right)\right) \\
& =v\left(\hat{\Pi}^{k-2}\left(\theta^{*}\right), 0\right) \hat{\Pi}^{\prime}\left(\Pi^{k-2}\left(\theta^{*}\right)\right) \hat{\Pi}^{\prime}\left(\Pi^{k-1}\left(\theta^{*}\right)\right) \\
& \vdots \\
& =v\left(\theta^{*}, 0\right) \prod_{j=0}^{k-1}\left(\hat{\Pi}^{\prime} \circ \hat{\Pi}^{j}\right)\left(\theta^{*}\right) .
\end{aligned}
$$

So Eq. (11) implies that $v\left(\theta^{*}, 0\right)=0$ because $\hat{\Pi}^{\prime} \circ \hat{\Pi}^{j}>0$ for all $j \in \mathbb{N}$ by (10). Hence $v$ (and also $\hat{v}$ ) vanishes on the periodic orbit through the point $\left(\theta^{*}, 0\right)$.

\section{B. The restriction of inverse Jacobi multipliers to invariant tori or spheres}

We consider a two-dimensional manifold $\Gamma$ embedded in $\mathbb{R}^{3}$ that can be a torus $\mathbb{T}^{2}$ or a sphere $\mathbb{S}^{2}$. In any case, there are local coordinates $(\rho, \theta, \varphi)$ with $\rho \in \mathbb{R}^{+}$and $(\theta, \varphi) \in \mathbb{T}^{2}$ or $(\theta, \varphi) \in \mathbb{S}^{2}$ such that the equation of $\Gamma$ is given by $\Gamma=\{\rho=r\}$. In the case of a torus, these coordinates are (4) while for the sphere they are the spherical coordinates $x=\rho \cos \varphi \sin \theta, y=\rho \sin \varphi \sin \theta$, and $z=\rho \cos \theta$.

Proposition 9: Any analytic vector field $\mathcal{X}$ in $\mathbb{R}^{3}$ with the invariant manifold $\Gamma=\{\rho=r\}$ written in the local curvilinear coordinates $(\rho, \theta, \varphi)$ is expressed as

$$
\begin{aligned}
& \dot{\rho}=F^{\kappa}(\rho) f_{1}(\rho, \theta, \varphi), \\
& \dot{\theta}=f_{2}(\rho, \theta, \varphi), \\
& \dot{\varphi}=f_{3}(\rho, \theta, \varphi),
\end{aligned}
$$

with $F(\rho)=r-\rho, f_{i}$ analytic functions near $\Gamma, \kappa \in \mathbb{Z}^{+}$, and $\left.f_{1}\right|_{\Gamma} \not \equiv 0$. Under the extra hypothesis that $\left.f_{1}\right|_{\Gamma}$ be sign-defined, $\Gamma$ is attractor or repelling when $\kappa$ is odd and $\left.f_{1}\right|_{\Gamma}$ is positive or negative, respectively. Otherwise, $\Gamma$ is semi-stable when $\kappa$ is even. Let $V$ be an analytic inverse Jacobi multiplier of (12) with $\Gamma \subset V^{-1}(0)$ having vanishing multiplicity $m \in \mathbb{Z}^{+}$, that is, $V=F^{m} W$ with $\left.W\right|_{\Gamma} \not \equiv 0$. Then, $\left.W\right|_{\Gamma}$ is an inverse Jacobi multiplier of the restricted vector field $\left.\mathcal{X}\right|_{\Gamma}$ provided that either $\kappa=m=1$ or $\kappa \geq 2$.

Proof. The invariance of $\Gamma$ and the analyticity of system (12) implies that $\dot{\rho}=F^{\kappa}(\rho) f_{1}(\rho, \theta, \varphi)$ and that there is $\kappa \in \mathbb{Z}^{+}$such that $\left.f_{1}\right|_{\Gamma} \not \equiv 0$. Imposing the restriction $\left.f_{1}\right|_{\Gamma}$ to be sign-defined we reach the conclusions on the attractivity, repulsivity, or semi-stability of $\Gamma$ just by simple inspection of the first component of system (12).

Imposing that $V=F^{m} W$ satisfies the partial differential equation $\mathcal{X}(V)=V \operatorname{div}(\mathcal{X})$ and taking the restriction of this equation to $\Gamma$ it is straightforward to see that

$$
\mathcal{Y}(v)=v \operatorname{div}(\mathcal{Y})+G,
$$

where $\mathcal{Y}=\left.\mathcal{X}\right|_{\Gamma}, v=\left.W\right|_{\Gamma}$ and $G=\left.(\kappa-m) f_{1}\right|_{\Gamma} F^{\kappa-1}$ Therefore, $\left.G\right|_{\Gamma}=0$ when either $\kappa=m=1$ or $\kappa \geq 2$ and in that case $v$ is an inverse Jacobi multiplier of $\mathcal{Y}$.

Remark 10: The case $m=0$ can also be added in the proof of Proposition 9. The outcome is that if $V$ is an inverse Jacobi multiplier of the vector field $\mathcal{X}$ associated to system (12) with $\Gamma \not \subset V^{-1}(0)$, then 
$\left.V\right|_{\Gamma}$ is an inverse Jacobi multiplier of the restricted vector field $\left.\mathcal{X}\right|_{\Gamma}$ provided that $\kappa \geq 2$.

Remark 11: In the proof of Proposition 9, we check that $\mathcal{X}(F)=K F$ with cofactor $K=-F^{\kappa-1} f_{1}$. Therefore, $\left.K\right|_{\Gamma}<0$ if $\kappa=1$ and $\left.f_{1}\right|_{\Gamma}>0$, in which case, from the continuity of $K, K$ is negative in a neighborhood $U \subset \mathbb{R}^{3}$ of $\Gamma$. So only under these restrictions we can apply Theorem 1 with the open set $\Omega=U \backslash \Gamma$ to conclude that $\Gamma$ is an attractor.

As a corollary of Theorem 8, Proposition 9, and Remark 10, we obtain a complementary result to Proposition 6.

Corollary 12: Consider an analytic vector field $\mathcal{X}$ in $\mathbb{R}^{3}$ having the invariant torus $\mathbb{T}^{2}=\{\rho=r\}$ expressed in local curvilinear coordinates (4) like (12) with $\left.f_{1}\right|_{\mathbb{T}^{2}}$ sign-defined. Assume $\left.\mathcal{X}\right|_{\mathbb{T}^{2}}$ has a cross section $\mathbb{S}^{1}$ and a rational rotation number. Let $V$ be an analytic inverse Jacobi multiplier of system (12).

(i) If $\mathbb{T}^{2} \subset V^{-1}(0)$ with vanishing multiplicity $m \in \mathbb{Z}^{+}$and either $\kappa=m=1$ or $\kappa \geq 2$, then $W=V /(r-\rho)^{m}$ restricted to $\mathbb{T}^{2}$ is an inverse Jacobi multiplier of $\left.\mathcal{X}\right|_{\mathbb{T}^{2}}$ with $\left.W\right|_{\mathbb{T}^{2}} \not \equiv 0$, and $\left.W\right|_{\mathbb{T}^{2}}$ vanishes in all the periodic orbits on $\mathbb{T}^{2}$.

(ii) If $\mathbb{T}^{2} \not \subset V^{-1}(0)$ and $\kappa \geq 2$, then $\left.V\right|_{\mathbb{T}^{2}}$ is an inverse Jacobi multiplier of $\left.\mathcal{X}\right|_{\mathbb{T}^{2}}$, and $\left.V\right|_{\mathbb{T}^{2}}$ vanishes in all the periodic orbits on $\mathbb{T}^{2}$.

\section{EXAMPLES}

A simple method to construct vector fields $\mathcal{X}$ in $\mathbb{R}^{n}$ possessing a prescribed invariant manifold $\{F=0\} \subset \mathbb{R}^{n}$ is given by $\mathcal{X}=\mathcal{X}_{F}+F \mathcal{Y}$, where $\mathcal{Y}$ is an arbitrary vector field and $\mathcal{X}_{F}$ is a vector field having $F$ as first integral. For example, in $\mathbb{R}^{3}$ we can take $\mathcal{X}_{F}=\mathcal{Z} \times \nabla F$, where $\mathcal{Z}$ is an arbitrary vector field in $\mathbb{R}^{3}$. Since $\mathcal{X}_{F}(F) \equiv 0$ we have $\mathcal{X}(F)=K F$, where the cofactor $K$ of $F$ for $\mathcal{X}$ is just $K=\mathcal{Y}(F)$. Since $\mathcal{Y}$ is arbitrary, we may choose it in order that $K$ be semi-definite negative with the simple selection $\mathcal{Y}=-\nabla F$, which produces $K=-\|\nabla F\|^{2}$, hence $K$ restricted to $\mathbb{R}^{n} \backslash \operatorname{crit}(F)$ is negative. Here, $\operatorname{crit}(F)$ denotes the set of critical points of the function $F$. This construction was used in Ref. 9, for example. If additionally we take $\mathcal{X}_{F}$ divergence free, that is, $\operatorname{div}\left(\mathcal{X}_{F}\right) \equiv 0$, then it is easy to check that $F$ is an inverse Jacobi last multiplier if and only if it is a harmonic function, that is, it is a solution of the Laplace equation $\nabla^{2} F=0$.

Of course, the topology of the manifold $\{F=0\}$ imposes some restrictions on the behavior of the flow on it. In 1964 Schwartz, ${ }^{16}$ generalizing Denjoy's theorem, proved that the only minimal sets that a $C^{2}$ - flow on a compact surface can have are equilibria, periodic orbits, or the whole surface (in which case the surface is a torus).

\section{A. The invariant sphere}

Example 1: We consider the sphere $\mathbb{S}^{2}$ of radius $R$ embedded in $\mathbb{R}^{3}$ with equation $\left\{F(x, y, z)=x^{2}+y^{2}+z^{2}-R^{2}=0\right\}$. Taking the polynomial vector field in $\mathbb{R}^{3}$ given by $\mathcal{X}=\mathcal{Z} \times \nabla F-F \nabla F$ with a nonzero constant vector field $\mathcal{Z}=Z_{1} \partial_{x}+Z_{2} \partial_{y}+Z_{3} \partial_{z}$. The cofactor of $F$ is $K(x, y, z)=-4\left(x^{2}+y^{2}+z^{2}\right)$, which is negative in $\mathbb{R}^{3} \backslash\{(0,0,0)\}$. The singularities of $\mathcal{X}$ are located at the origin with eigenvalues $2 R^{2}$ and $2\left(R^{2} \pm i\|\mathcal{Z}\|\right)$, and hence it is a source of flow, and at the points $\pm R \mathcal{Z} /\|\mathcal{Z}\|$ on $\mathbb{S}^{2}$.

We claim that $\mathbb{S}^{2}$ is an attractor set with the basin of attraction $\mathbb{R}^{3} \backslash\left\{\mathbb{S}^{2} \cup\{(0,0,0)\}\right\}$. To see this, we consider a neighborhood
$U$ of $\mathbb{S}^{2}$ whose boundary is given by two spheres $\left\{G_{j}=0\right\}$ centered at the origin of arbitrary radius $R_{1}$ and $R_{2}$ satisfying $0<R_{1}$ $<R<R_{2}$, that is, $G_{j}=x^{2}+y^{2}+z^{2}-R_{j}^{2}$. We have $\mathcal{X}\left(G_{j}\right)(x, y, z)$ $=-4\left(x^{2}+y^{2}+z^{2}\right) F(x, y, z)$ so that $\left.\mathcal{X}\left(G_{j}\right)\right|_{\left\{G_{j}=0\right\}}=-4 R_{j}^{2}\left(R_{j}^{2}-R^{2}\right)$, hence $\left.\mathcal{X}\left(G_{1}\right)\right|_{\left\{G_{1}=0\right\}}>0$ and $\left.\mathcal{X}\left(G_{2}\right)\right|_{\left\{G_{2}=0\right\}}<0$ implying that $U$ is a trapping neighborhood of $\mathbb{S}^{2}$ and the claim follows. Notice that we can apply Theorem 1 with $\Omega=U \backslash \mathbb{S}^{2}$.

On the other hand, it is straightforward to see that $V(x, y, z)$ $=\left(x^{2}+y^{2}+z^{2}\right)^{3 / 2} F(x, y, z)$ is an inverse Jacobi multiplier of $\mathcal{X}$ and $\mathbb{S}^{2} \subset V^{-1}(0)$. The restricted vector field $\left.\mathcal{X}\right|_{\mathbb{S}^{2}}=\mathcal{Z} \times \nabla F$ is linear and has two polynomial independent first integrals: $F$ and $\mathcal{Z}(F)$. Therefore, $\mathcal{X}$ induces on $\mathbb{S}^{2}$ a foliation of periodic orbits, which are indeed circles around an axis in the direction $\mathcal{Z}$.

Example 2: Take the vector field $\mathcal{X}=\mathcal{Z} \times \nabla F-F \nabla F$ with $\operatorname{div}(\mathcal{Z} \times \nabla F) \equiv 0$ and consider the harmonic function

$$
F(x, y, z)=\frac{1}{\sqrt{x^{2}+y^{2}+z^{2}}}-\frac{1}{R},
$$

both defined in $D=\mathbb{R}^{3} \backslash\{(0,0,0)\}$ such that $\mathbb{S}^{2}=F^{-1}(0)$. By construction, $F$ is an inverse Jacobi multiplier of the vector field $\mathcal{X}$ in $D$ and $\mathbb{S}^{2}$ is an invariant set.

To be more precise, we can take $\mathcal{Z}=Z_{1} \partial_{x}+Z_{2} \partial_{y}+Z_{3}(z) \partial_{z}$ with constants $Z_{1}$ and $Z_{2}$ in which case $\operatorname{div}(\mathcal{X})=-\left(x^{2}+y^{2}+z^{2}\right)^{-2}$ is negative in $D$ but $\mathbb{S}^{2}$ is not an attractor set because $D$ is not simply connected and the origin acts like a sink under the flow of $\mathcal{X}$. Compare this example in the light of Remark 4 on Theorem 1 with the selection $\Omega=\mathbb{R}^{3} \backslash\left\{\mathbb{S}^{2} \cup\{0,0,0\}\right\}$ that satisfies $\phi_{t}(\Omega)=\Omega$ but the cofactor $K=\operatorname{div}(\mathcal{X})$ of $F$ is not uniformly bounded in $\Omega$. Proceeding in the same way as in the former example we consider the same two spheres $\left\{G_{j}=0\right\}$ of the previous Example 1 and we obtain

$$
\left.\mathcal{X}\left(G_{j}\right)\right|_{\left\{G_{j}=0\right\}}=\frac{2}{R_{j}}\left(\frac{1}{R_{j}}-\frac{1}{R}\right),
$$

where $0<R_{1}<R<R_{2}$. Therefore, $\left.\mathcal{X}\left(G_{1}\right)\right|_{\left\{G_{1}=0\right\}}>0$ and $\left.\mathcal{X}\left(G_{2}\right)\right|_{\left\{G_{2}=0\right\}}<0$, implying that $\mathbb{S}^{2}$ is semi-stable (repelling the interior and attracting the exterior).

See the flow near the sphere in Fig. 2 for the particular case $\mathcal{Z}=\partial_{x}+\partial_{y}$.

\section{B. An attractor torus with limit cycles}

Using the curvilinear coordinates $(\rho, \theta, \varphi)$ defined by (4), we consider the differential system

$$
\begin{aligned}
& \dot{\rho}=r-\rho, \\
& \dot{\theta}=1-k \sin (\theta-\varphi), \\
& \dot{\varphi}=\omega+k \sin (\theta-\varphi),
\end{aligned}
$$

which arises in the studies of linearly coupled limit cycle oscillators with parameters $0 \leq \omega \leq 1$ and $k \geq 0$, see Ref. 10 . From the first component, we see that (13) possesses $\mathbb{T}^{2}$ as an invariant attractor set. The last two components of (13) define a flow on $\mathbb{T}^{2}$ whose behavior is easily obtained from the associated equation $\dot{\Omega}=1$ $-\omega-2 k \sin \Omega$, where $\Omega=\theta-\varphi$. The equilibria of this equation produce phase-locked periodic orbits in (13). Since it can be solved explicitely, it is easy to show that, under the parameter constraint 


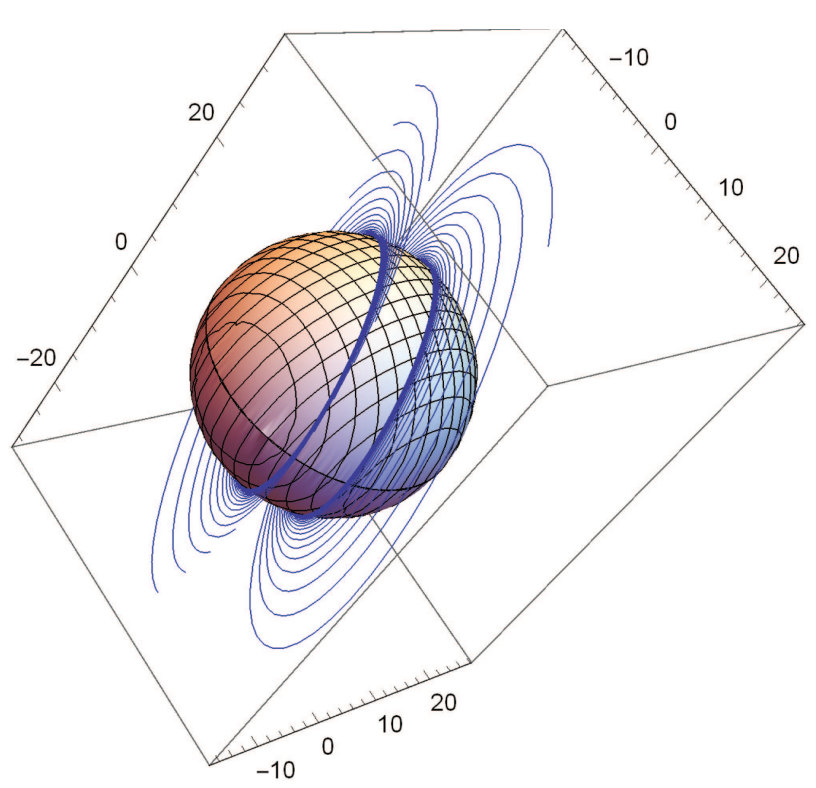

FIG. 2. Two orbits attracted by two different periodic orbits on the sphere.

$2 k>1-\omega$, there are two isolated hyperbolic periodic orbits (one stable and the other unstable) of system (13), with $\dot{\theta}=\dot{\varphi}=\omega^{*}$ being the constant frequency $\omega^{*}=(\omega+1) / 2$. Both phase-locked periodic orbits lie on the curve $\mathcal{C}=\left\{(\theta, \varphi) \in \mathbb{T}^{2}: \mathcal{F}(\theta, \varphi)=\omega-1\right.$ $+2 k \sin (\theta-\varphi)=0\}$. A saddle-node bifurcation occurs at $2 k=1$ $-\omega$ when both periodic orbits coalesce and disappear for $2 k<1-\omega$ giving (structurally unstable) either quasiperiodic or rational flow depending on the parameters, see the forthcoming analysis using rotation numbers to discern between these two possibilities. Let $\Sigma=\{(\theta, \varphi): \theta=0\} \subset \mathbb{T}^{2}$ be a cross section for the flow of family (13) on $\mathbb{T}^{2}$ and consider its Poincaré map $\Pi: \mathbb{S}^{1} \rightarrow \mathbb{S}^{1}$. In Ref. 10 , the rotation number $\Pi$ is computed when $\omega \in[0,1]$ is fixed and the parameter $k \geq 0$. The outcome is that $\operatorname{Rot}(\Pi)=1$ for $2 k \geq 1-\omega$ whereas

$$
\operatorname{Rot}(\Pi)=\frac{1+\omega-\delta(\omega, k)}{1+\omega+\delta(\omega, k)}, \text { with } \delta(\omega, k)=\sqrt{\left(1-\omega^{2}\right)^{2}-4 k^{2}},
$$

when $2 k<1-\omega$. The function $\operatorname{Rot}(\Pi)$ is a continuous increasing function of $k$ whose values are in the interval $[\omega, 1]$. In summary, when $2 k>1-\omega$, there are exactly two isolated hyperbolic periodic orbits to which all the other orbits on $\mathbb{T}^{2}$ approach in the future or in the past. At $2 k=1-\omega$, a saddle-node bifurcation occurs and the former two periodic orbits coalesce in a semistable periodic orbit. Finally, in the complementary case $2 k<1-\omega$, all the orbits on $\mathbb{T}^{2}$ are periodic or asymptotically periodic if $\operatorname{Rot}(\Pi) \in \mathbb{Q}$, while the dynamics is quasi-periodic where the orbits are dense and nonperiodic for $\operatorname{Rot}(\Pi) \notin \mathbb{Q}$.

\section{The case $\omega=1$}

For any $k \geq 0$, there are two hyperbolic periodic orbits on $\mathcal{C}=\left\{(\theta, \varphi) \in \mathbb{T}^{2}: \mathcal{F}(\theta, \varphi)=\sin (\theta-\varphi)=0\right\}$. Let $U_{\mathbb{T}^{2}}$ be a

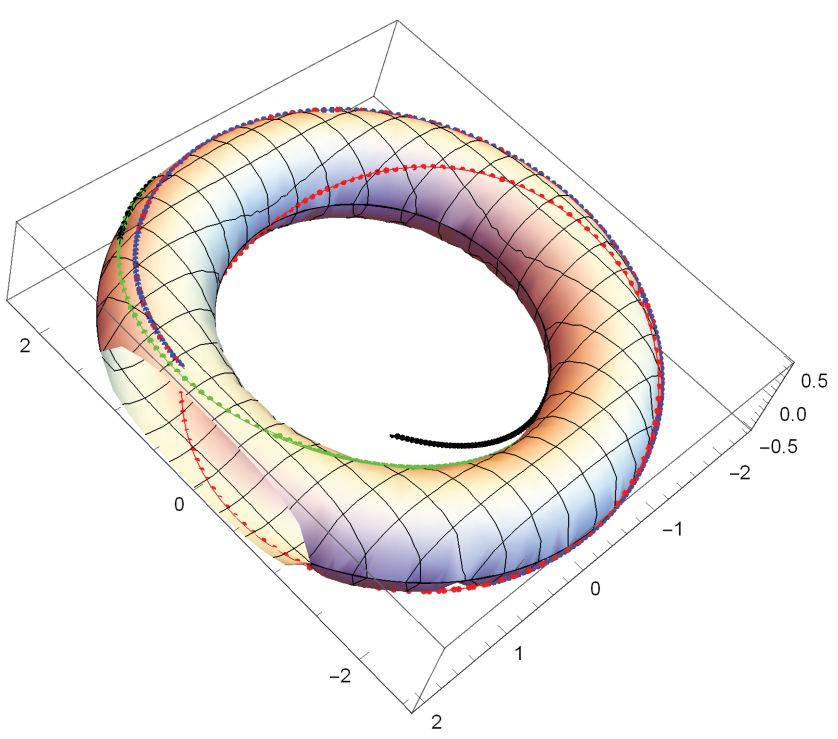

FIG. 3. Two limit cycles (red and green orbits) on the invariant attractor torus and the other two orbits approaching them.

sufficiently small neighborhood of $\mathbb{T}^{2}$ and consider the following two functions $V_{i}: U_{\mathbb{T}^{2}} \rightarrow \mathbb{R}$ with $V_{i} \in C^{1}\left(U_{\mathbb{T}^{2}}\right)$ for $i=1,2$ : $V_{1}(\rho, \theta, \varphi)=(r-\rho) \mathcal{F}(\theta, \varphi)$ and

$$
V_{2}(\rho, \theta, \varphi)= \begin{cases}\mid \frac{\mathcal{F}(\theta, \varphi)}{\sqrt{\tan \left(\frac{\theta-\varphi}{2}\right)} \mid} \quad \text { if } \quad \theta-\varphi \notin\{0, \pi\}, \\ 0 & \text { if } \theta-\varphi \in\{0, \pi\},\end{cases}
$$

where the bars denote the modulus of the complex number inside. We claim that $V_{1}$ and $V_{2}$ are both inverse Jacobi multipliers (this may be checked by direct computations) defined on $U_{\mathbb{T}^{2}}$ such that $\mathbb{T}^{2} \subset V_{1}^{-1}(0), \mathbb{T}^{2} \not \subset V_{2}^{-1}(0)$ but the condition $\left(\mathbb{T}^{2} \cap \mathcal{C}\right) \subset V_{2}^{-1}(0)$ holds.

We draw the flow near the torus in Fig. 3 for the parameters $\omega=k=1$ and the torus with radius $R=2$ and $r=1 / 2$.

\section{A non-attractor invariant torus in $\boldsymbol{V}^{-1}(0) \subset \mathbb{R}^{3}$}

The torus $\mathbb{T}^{2}$ can be embedded in $\mathbb{R}^{3}$ as follows: the points $(x, y, z) \in \mathbb{T}^{2}$ are parameterized by

$$
x=(R+r \cos \theta) \cos \varphi, \quad y=(R+r \cos \theta) \sin \varphi, z=r \sin \theta .
$$

Assuming a dynamics $(\theta(t), \varphi(t))$ on $\mathbb{T}^{2}$, we have differentiating that

$$
\begin{aligned}
& \dot{x}=-r \cos \varphi \sin \theta \dot{\theta}-(R+r \cos \theta) \sin \varphi \dot{\varphi}, \\
& \dot{y}=-r \sin \varphi \sin \theta \dot{\theta}-(R+r \cos \theta) \cos \varphi \dot{\varphi}, \\
& \dot{z}=r \cos \theta \dot{\theta},
\end{aligned}
$$


which can be rewritten as

$$
\begin{aligned}
& \dot{x}=-y \dot{\varphi}-\frac{2 R x z}{R^{2}-r^{2}+x^{2}+y^{2}+z^{2}} \dot{\theta}, \\
& \dot{y}=x \dot{\varphi}-\frac{2 R y z}{R^{2}-r^{2}+x^{2}+y^{2}+z^{2}} \dot{\theta}, \\
& \dot{z}=\frac{1}{2 R}\left(-r^{2}-R^{2}+x^{2}+y^{2}+z^{2}\right) \dot{\theta} .
\end{aligned}
$$

Since $R^{2}-r^{2}+x^{2}+y^{2}+z^{2}>0$ in all $\mathbb{R}^{3}$, we perform a time rescaling and obtain the vector field

$$
\begin{aligned}
\dot{x}= & -y\left(R^{2}-r^{2}+x^{2}+y^{2}+z^{2}\right) \dot{\varphi}-2 R x z \dot{\theta}, \\
\dot{y}= & x\left(R^{2}-r^{2}+x^{2}+y^{2}+z^{2}\right) \dot{\varphi}-2 R y z \dot{\theta}, \\
\dot{z}= & \frac{1}{2 R}\left(R^{2}-r^{2}+x^{2}+y^{2}+z^{2}\right) \\
& \times\left(-r^{2}-R^{2}+x^{2}+y^{2}+z^{2}\right) \dot{\theta},
\end{aligned}
$$

which has, by construction, the invariant torus $\mathbb{T}^{2}$. Taking a linear flow on the torus $(\dot{\theta}, \dot{\varphi})=\left(\omega_{1}, \omega_{2}\right)$ with rationally independent frequencies $\omega_{1} / \omega_{2} \notin \mathbb{Q}$ implies that all the orbits on $\mathbb{T}^{2}$ are dense. Otherwise, when $\omega_{1} / \omega_{2} \in \mathbb{Q}$ all the orbits on $\mathbb{T}^{2}$ are periodic.

One can easily check that neither the cofactor $K$ of $F$ nor $\operatorname{div}(\mathcal{X})$ is sign defined in any neighborhood of $\mathbb{T}^{2}$. Consider anyway a neighborhood $U\left(r^{*}\right) \subset \mathbb{R}^{3}$ of $\mathbb{T}^{2}$ whose boundary is formed by the concentric tori centered at the origin with common generating radius $R$ and tube radius $r_{*}-r$ and $r^{*}+r$. Since $U(0)=\mathbb{T}^{2}$, we have that $U\left(r^{*}\right)$ is as small as we want by taking $r^{*}>0$ sufficiently close to zero. We can parameterize $U\left(r^{*}\right)$ as $U\left(r^{*}\right)=\left\{((R+\rho \cos \theta) \cos \varphi,(R+\rho \cos \theta) \sin \varphi, \rho \sin \theta) \in \mathbb{R}^{3}\right.$ : $\left.(\theta, \varphi) \in \mathbb{S}^{1} \times \mathbb{S}^{1}, r-r^{*} \leq \rho \leq r+r_{*}\right\}$, and the volume element is $d x d y d z=J d \rho d \theta d \varphi$ with Jacobian $J=\rho(R+\rho \cos \theta)$, hence the restriction $\left.J\right|_{\mathbb{T}^{2}} \neq 0$. The divergence of (14) is

$$
\begin{aligned}
\operatorname{div}(\mathcal{X}) & =\frac{2 \omega_{1}}{R} z\left(x^{2}+y^{2}+z^{2}-r^{2}-2 R^{2}\right) \\
& =\frac{2 \omega_{1}}{R} \rho\left(\rho^{2}+2 R \rho \cos \theta-r^{2}-R^{2}\right) \sin \theta .
\end{aligned}
$$

Then, the volume integral of $\operatorname{div}(\mathcal{X})$ on $U\left(r^{*}\right)$ is given by

$$
\begin{aligned}
& \int_{U\left(r^{*}\right)} \operatorname{div}(\mathcal{X}) d x d y d z \\
& =\int_{0}^{2 \pi} d \varphi \int_{0}^{2 \pi} d \theta \int_{r-r^{*}}^{r+r_{*}} \operatorname{div}(\mathcal{X}) \rho(R+\rho \cos \theta) d \rho=0
\end{aligned}
$$

for any $r^{*} \geq 0$. Therefore, by (1), $\left|\phi_{t}\left(U\left(r^{*}\right)\right)\right|$ is constant for all $t$ and any $r^{*}$, which implies that $\mathbb{T}^{2}$ is not an attractor set.

Taking squares to remove the involved square root, the equation $F=0$ can be rewritten into the polynomial form $\mathcal{F}(x, y, z)=0$ with $\mathcal{F}(x, y, z)=4 R^{2}\left(x^{2}+y^{2}\right)-\left(-r^{2}+R^{2}+x^{2}\right.$ $\left.+y^{2}+z^{2}\right)^{2}$ and obviously $\mathbb{T}^{2} \subset\{\mathcal{F}=0\}$, which is an invariant algebraic surface of (14). Moreover, the invariance of the $z$-axis for the flow of (14) is reflected by the fact that $\left\{x^{2}+y^{2}=0\right\}$ is also invariant. Now, we can construct the following inverse Jacobi multiplier $V(x, y, z)=\left(x^{2}+y^{2}\right)^{1 / 2} \mathcal{F}(x, y, z)$ and $\mathbb{T}^{2} \subset V^{-1}(0)$.

\section{Higher dimensional flows}

We cannot use $n$ degree of freedom integrable Hamiltonian system giving rise to flows on $n$-dimensional tori since they are not attractor sets. Instead, we will consider the flow generated by uncoupled nonconservative oscillators possessing attracting limit cycles:

$$
\dot{x}_{i}=-\omega_{i} y_{i}-x_{i} f\left(x_{i}, y_{i}\right), \quad \dot{y}_{i}=\omega_{i} x_{i}-y_{i} f\left(x_{i}, y_{i}\right), \quad i=1,2,
$$

with $f\left(x_{i}, y_{i}\right)=x_{i}^{2}+y_{i}^{2}-1$ and constant frequencies $\omega_{i}$. The product $\mathbb{S}^{1} \times \mathbb{S}^{1}$ of the two limit cycles (circles $\left\{f\left(x_{i}, y_{i}\right)=0\right\}$ of radius 1 ) in the $\left(x_{1}, y_{1}\right)$ and $\left(x_{2}, y_{2}\right)$ planes is an attractor two-torus $\mathbb{T}^{2} \subset \mathbb{R}^{4}$. Taking polar coordinates $x_{i}=r_{i} \cos \varphi_{i}, y_{i}=r_{i} \sin \varphi_{i}$, system (15) writes

$$
\dot{r}_{i}=r_{i}\left(1-r_{i}^{2}\right), \quad \dot{\varphi}_{i}=\omega_{i}, \quad i=1,2 .
$$

Thus, $\mathbb{T}^{2}=\left\{r_{1}=r_{2}=1\right\}$ and its points are parameterized by the angles $\left(\varphi_{1}, \varphi_{2}\right)$. It is easy to see that $V\left(r_{1}, \varphi_{1}, r_{2}, \varphi_{2}\right)=\prod_{i=1}^{2} r_{i}\left(r_{i}^{2}-1\right)$ is an inverse Jacobi multiplier of (16) and that $\mathbb{T}^{2} \subset V^{-1}(0)$. Moreover, we claim that (16) does not admit any $C^{1}$ function on $\mathbb{T}^{2}$ as first integral, because (15) cannot have a first integral in a neighborhood of the limit cycles $f\left(x_{i}, y_{i}\right)=0$. In particular, this implies that $V$ is, up to multiplicative constants, the only inverse Jacobi multiplier of (16).

Remark 13: Since (16) has separated variables one can easily obtain two functions $H_{i}\left(r_{i}, \varphi_{i}\right)=r_{i}^{-2}\left(r_{i}^{2}-1\right) \exp \left(2 \varphi_{i} / \omega_{i}\right)$ with $i=1,2$ that satisfy $\mathcal{X}\left(H_{i}\right) \equiv 0, \mathcal{X}$ being the vector field associated with (16), but they are not periodic functions on $\varphi_{i}$ so do not define functions on $\mathbb{T}^{2}$.

\section{ACKNOWLEDGMENTS}

I.A.G., J.G., and S.M. are partially supported by Ministerio de Ciencia, Innovación y Universidades under Grant No. MTM201784383-P and by AGAUR under Grant No. 2017SGR-1276. J.L. is partially supported by Ministerio de Ciencia, Innovación y Universidades, Agencia Estatal de Investigación under Grant No. MTM201677278-P (FEDER), the Agència de Gestió d'Ajuts Universitaris i de Recerca under Grant No. 2017SGR1617, and the H2020 European Research Council under Grant No. MSCA-RISE-2017-777911.

\section{APPENDIX: LIOUVILLE'S FORMULA}

The following Liouville's formula is a well-known result about the divergence-flow relation, see the proof in Ref. 11.

Proposition 14: Let $U \subset \mathbb{R}^{n}$ be any measurable subset and $\phi_{t}$ be the flow of a $C^{1}$ differential system with associated vector field $\mathcal{X}$ defined in $\mathbb{R}^{n}$. Then,

$$
\left.\frac{d}{d t}\left|\phi_{t}(U)\right|\right|_{t=t_{0}}=\int_{\phi_{t_{0}}(U)} \operatorname{div}(\mathcal{X}) d x .
$$

\section{DATA AVAILABILITY}

The data that support the findings of this study are available within the article. 


\section{REFERENCES}

${ }^{1}$ L. R. Berrone and H. Giacomini, "Inverse Jacobi multipliers," Rend. Circ. Mat. Palermo (2) 52, 77-130 (2003).

${ }^{2}$ A. Buică and I. A. García, "Inverse Jacobi multipliers and first integrals for nonautonomous differential systems," Z. Angew. Math. Phys. 66, 573-585 (2015). ${ }^{3}$ A. Buică, I. A. García, and S. Maza, "Inverse Jacobi multipliers: Recent applications in dynamical systems," in Progress and Challenges in Dynamical Systems, Springer Proceedings in Mathematics \& Statistics (Springer, 2013), Vol. 54, pp. $127-141$

${ }^{4} \mathrm{~A}$. Enciso and D. Peralta-Salas, "Existence and vanishing set of inverse integrating factors for analytic vector fields," Bull. Lond. Math. Soc. 41, 1112-1124 (2009).

${ }^{5}$ G. B. Folland, Introduction to Partial Differential Equations (Princeton University Press, 1995).

${ }^{6}$ I. A. García, H. Giacomini, and M. Grau, "The inverse integrating factor and the Poincaré map," Trans. Am. Math. Soc. 362, 3591-3612 (2010).

${ }^{7}$ I. A. García and M. Grau, "A survey on the inverse integrating factor," Qual. Theor. Dyn. Syst. 9, 115-166 (2010).
${ }^{8} \mathrm{H}$. Giacomini, J. Llibre, and M. Viano, "On the nonexistence, existence, and uniqueness of limit cycles," Nonlinearity 9, 501-516 (1996).

${ }^{9} \mathrm{~F}$. González-Gascón and D. Peralta-Salas, "Attractors and symmetries of vector fields: The inverse problem," J. Math. Anal. Appl. 335, 789-807 (2007).

${ }^{10} \mathrm{~J}$. Guckenheimer and P. Holmes, Nonlinear Oscillations, Dynamical Systems, and Bifurcations of Vector Fields, Applied Mathematical Sciences (SpringerVerlag, New York, 1990), Vol. 42.

${ }^{11} \mathrm{P}$. Hartman, Ordinary differential equations, corrected reprint of the second (1982) edition (Birkhäuser, Boston, 2002).

${ }^{12}$ G. Leonov, "Strange attractors and classical stability theory," Nonlinear Dyn. Syst. Theor. 8, 49-96 (2008), available at http://www.e-ndst.kiev.ua/v8n1.htm

${ }^{13}$ J. Milnor, "On the concept of attractor," Commun. Math. Phys. 99, 177-195 (1985).

${ }^{14} \mathrm{~J}$. Milnor, "On the concept of attractor: Correction and remarks," Commun. Math. Phys. 102, 517-519 (1985).

${ }^{15} \mathrm{I}$. Nikolaev and E. Zhuzhoma, Flows on 2-Dimensional Manifolds. An Overview, Lecture Notes in Mathematics (Springer-Verlag, Berlin, 1999), Vol. 1705.

${ }^{16} \mathrm{~A}$. Schwartz, "A generalization of the Poincaré-Bendixson theorem to closed two-dimensional manifolds," Am. J. Math. 85, 453-458 (1963). 